\title{
The Geometry of Projective Blending Surfaces*
}

\author{
Christoph Hoffmann \\ John Hopcroft
}

TR86-758

May 1986

Department of Computer Science Cornell University

Ithaca, NY 14853

* Supported in part by National Science Foundation Grants ECS 83-12096, DCR85-02568, and DCR 85-12443. 


\title{
The Geometry of Projective Blending Surfaces ${ }^{1}$
}

\author{
Christoph Hoffmann \\ Computer Science Department \\ Purdue University \\ West Lafayette, Indiana 47907 \\ John Hopcroft \\ Department of Computer Science \\ Cornell University \\ Ithaca, New York 14853
}

\begin{abstract}
Blending surfaces smoothly join two or more primary surfaces that otherwise would intersect in edges. We outline the potential method for deriving blending surfaces, and explain why the method needs to be considered in projective parameter space, concentrating on the case of blending quadrics. Let $W$ be the quadratic polynomial substituted for the homogenizing variable of parameter space. We show that a blending surface derived in projective parameter space is the projective image of a different blending surface derived in affine parameter space, provided that $W=U^{2}$ for some linear $U$. All blending surfaces may therefore be classified on basis of the projective classification of $W$.
\end{abstract}

1 Supported in part by National Science Foundation Grants ECS 83-12096, DCR 85-02568, and DCR 85-12443. 


\section{The Geometry of Projective Blending Surfaces}

\section{Introduction}

In computer-aided design, geometric models of objects in manufacture are created and manipulated. Virtually all manufactured solid objects contain surfaces meant to round sharp edges or smoothly connect surface areas that otherwise would intersect in creases. These connecting surfaces are known as blending surfaces in the literature on solid modeling.

Traditional solid modeling systems have not been successful at incorporating blending surfaces, and only recently successful attempts have been made at identifying surface classes that can be used conveniently as blending surfaces [3-8]. In general, blending surfaces joining curved algebraic surfaces must have algebraic degree higher than 2, and extensive classifications of useful blending surface families are not widely available.

In a sequence of papers $[3,4,5]$, we have identified a class of blending surfaces derived by substitution into base polynomials, and have analyzed some of their geometric properties. Our principal derivation procedure is called the potential method and is capable of deriving in a standardized manner blending surfaces for any two intersecting algebraic surfaces. We have explored primarily the properties of blending surfaces when derived from a quadratic base polynomial, due to the practical importance of this case. Higher degree base polynomials could be used equally well and would permit higher order continuity between the blending surface and the primary surfaces connected by it.

When we introduced the potential method in [3], we used a quadratic base polynomial in which three free parameters had to be chosen in order to derive a specific surface. These parameters have a very intuitive interpretation: two control the relative distance of the curves at which the blending surface joins the primary surface, i.e., they control the width and centering of the blending surface about the intersection curve of the primary surfaces. The third parameter controls the shape of the blend. In [4] we have investigated the generality of this procedure, for blending quadrics. We found that in order to derive as general

a surface class as possible, the quadratic base polynomial must be homogeneous. That is, the underlying parameter space must be projective. However, this introduces additional parameters to be instantiated. The consequences of the additional choices for the resulting surface shapes are not fully understood. 
In this paper we summarize and extend our previous work on the properties of blending surfaces derived from homogeneous quadratic base polynomials. Our ultimate goal is to gain a comprehensive and intuitive interpretation of the parameters that must be instantiated in the base polynomial in order to derive specific blending surfaces. To this end, we characterize generic subclasses of our blending surfaces that are invariant under projective transformation.

The paper is structured as follows: After reviewing the necessary background and notation, we summarize in Section 3 the results of [4], leading to a succinct formulation of all quartic surfaces that serve as blending surfaces for two quadrics in general position. Then we review the potential method by considering first the affine formulation, marked by its intuitiveness, and subsequently the projective formulation. We then show the equivalence of the projective formulation for blending quadrics and the surfaces derivable by the methods of Section 3. In Section 6 we investigate how projective transformations map blending surfaces. In Section 7 we finally summarize the other methods proposed, and discuss the prospects for deriving blending surfaces automatically.

We do not explore techniques and configurations for blending corners that require joining more than two blends. From a theoretical perspective, this topic is exceedingly simple, for one could solve it by recursively blending the arising surfaces. There is, however, a practical matter: Proceeding systematically in this way, one quickly arrives at surfaces of very high algebraic degree, thereby seriously complicating the representation and manipulation of such surfaces. In [5] we have considered this problem and proposed practical solutions.

\section{Notation and Background}

Given two surfaces $S_{1}$ and $S_{2}$, a blending surface is any surface $S_{3}$ that is tangent to $S_{1}$ in a space curve $C_{1}$ and tangent to $S_{2}$ in a space curve $C_{2}$. All surfaces are considered in three dimensional $x, y, z$-space. For parts of our analysis, we must consider the surfaces in three dimensional complex projective space. It is our experience, however, that the intuitive content of the results we derive is not distorted by this needed generality. For example, we have never needed surface equations with complex coefficients.

We will consider blending algebraic surfaces of arbitrary degree. Such surfaces always have an implicit equation of the form $F=0$, where $F$ is a polynomial in $x, y$, and $z$. Note that some, but not all such surfaces may possess a parametric representation. For example, all quadric surfaces have a rational parameterization, but only some quartic 
surfaces can be so parameterized.

We must distinguish between the surface $S(F)$ with implicit equation $F=0$, and the polynomial $F$. The surface $S(F)$ consists of all points $(x, y, z)$ that are zeros of the polynomial $F$. The distinction is necessary so as to avoid confusing geometric and algebraic arguments.

We will consider algebraic curves and surfaces in other spaces as well. We call these spaces parameter spaces and consider mapping them to the $x, y, z$-space by substitution of polynomials in $x, y$, and $z$ for the principal parameter space coordinates. We use $r, s$, and $t$ as coordinates of affine parameter space, and $r, s, t$ and $u$ as coordinates of projective parameter space. These coordinates need to take on only real values. Polynomials in parameter spaces are denoted in lower case letters, e.g., $f, g, h \ldots$, and polynomials in $x, y, z-$ space by upper case letters, e.g., $F, G, H$....

\subsection{Algebraic Geometry}

In Section 3, methods from algebraic geometry are needed. This requires that we consider $F$ a polynomial over the ground field $\mathbf{C}$ of complex numbers. Unfortunately, the field $\mathbf{R}$ of reals is not algebraically closed, so most of the results of algebraic geometry no longer hold when only the real roots of $F$ are considered.

The set of all polynomials in $x, y, z$, and with real or complex coefficients is denoted $\mathbf{C}[x, y, z]$. The complete intersection of two surfaces $S(G)$ and $S(H)$ is denoted by $S(G, H)$, and is in general a space curve. Its algebraic description is provided by an ideal. Briefly, a set $I$ of polynomials is an ideal if, for all polynomials $G$ and $H$ of $I$, and an arbitrary polynomial $A$ in $\mathbf{C}[x, y, z]$, the polynomials $A G$ and $G+H$ are again in $I$. It is not hard to see that the curve $S(G, H)$ is described by the ideal $I=(G, H)$ generated by $G$ and $H$, that is, by the set of all polynomials of the form $A G+B H$, where $A$ and $B$ are polynomials in $\mathrm{C}[x, y, z]$.

Given an ideal $I$, the set of points at which all polynomials in $I$ vanish simultaneously is called the algebraic set of $I$ and is denoted $S(I)$. As we have mentioned, the algebraic set $S(G, H)$ of the ideal $(G, H)$ is in general a space curve, whereas the algebraic set $S(G)$ of the ideal $(G)$ generated by $G$ is a surface.

An ideal $I$ may have the property that for all product polynomials $A B$ in $I$ at least one of the factors is also in $I$. In that case $I$ is called a prime ideal. An algebraic set $S(I)$ is irreducible if it is not the union of two proper algebraic subsets. One knows that an algebraic set $S(I)$ is irreducible if and only if the ideal $I$ is prime (see, e.g., [2]). 
For example, a quadric surface is irreducible if it does not consist of two planes. The space curve $S(G, H)$ is irreducible if the curve does not have separate branches that individually are algebraic sets. Note that the two real branches of the hyperbola are not two separate algebraic sets, so that the hyperbola is irreducible. However, the intersection curve of two parallel circular cylinders has two lines each of which may be described separately as the intersection of a pair of planes, so that this intersection curve is reducible.

If $S(G, H)$ is a space curve and the surface $S(F)$ contains this curve, then there is a connection between the ideal $(G, H)$ and the polynomial $F$, given by

\section{Hilbert Nullstellensatz:}

If $S(F)$ contains $S(G, H)$, then there is an integer $k$ such that $F^{k}=A G+B H$. Moreover, if $S(G, H)$ is irreducible, then $k=1$.

\subsection{Projective Space}

The reader will be familiar with affine three dimensional space as the set of all points $(x, y, z)$ where $x, y$, and $z$ have values from a ground field, e.g., are real or complex numbers. Projective three dimensional space consists of all lines $(r x, r y, r z, r w)$ where $x, y, z$ and $w$ again have values from a ground field and $r \neq 0$. The case $x=y=z=w=0$ is excluded. By identifying the affine point $(x, y, z)$ with the projective line $(r x, r y, r z, r), r \neq 0$, three dimensional affine space is embedded into three dimensional projective space. Then the only additional "points" in projective space are lines of the form $(r x, r y, r z, 0)$ and are called points at infinity. The points at infinity form a plane called the plane at infinity.

If $F$ is a polynomial, $S(F)$ its algebraic set in affine space, then the corresponding set in projective space is the algebraic set of the associated homogeneous form $F_{0}$ of $F$. This form is obtained by substituting $x / w$ for $x, y / w$ for $y$, and $z / w$ for $z$, followed by multiplying with $w^{k}$ to eliminate the denominator. For example, if $F=x^{2}+y^{2}+z^{2}-2 x-9$, then its homogeneous form is $F_{0}=x^{2}+y^{2}+z^{2}-2 x w-9 w^{2}$.

In complex projective space a number of algebraic and geometric statements are true without exception. An example is the following standard result

\section{Theorem}

If $F$ is a homogeneous polynomial of degree $n$, then any line $L$ intersects $S(F)$ in $n$ points or is contained in $S(F)$.

In real affine space, $n$ is only an upper bound on the number of intersection points. The geometric degree of a surface $S(F)$ is the number of intersection points the surface has 
with a line $L$ in general position. The theorem states that the geometric degree of $S(F)$ is equal to the algebraic degree of $F$. The geometric degree of a space curve is the number of intersections with a plane in general position. An important result is the following theorem:

\section{Bezout's Theorem}

A surface $S(G)$ of degree $m$ and a surface $S(H)$ of degree $n$ intersect in a space curve of degree $m \cdot n$ or have a common component.

A projective transformation is a nonsingular linear transformation of projective space. Some projective transformations are familiar from computer graphics where they have been used to change stereographic projection to orthographic projection. Moreover, all nondegenerate conics may be projectively transformed into a circle of unit radius. See also Section 5 below.

When the matrix of the projective transformation is real-valued, then we speak of a real projective transformation, otherwise of a complex projective transformation. According to [1], the projective classification of all irreducible quadric surfaces under real projective transformation is as follows:

(1) The imaginary sphere $x^{2}+y^{2}+z^{2}+w^{2}$.

(2) The real sphere $x^{2}+y^{2}+z^{2}-w^{2}$. This class includes the ellipsoid, the two-sheeted hyperboloid, and the elliptic paraboloid.

(3) The one-sheeted hyperboloid $x^{2}+y^{2}-z^{2}-w^{2}$. This class includes also the hyperbolic paraboloid.

(4) The imaginary cylinder $x^{2}+y^{2}+w^{2}$.

(5) The real cylinder $x^{2}+y^{2}-w^{2}$. This class includes all conic cylinders and cones.

Under complex projective transformation the classes (1), (2) and (3) are no longer distinct, as well as the classes (4) and (5).

\section{A Completeness Theorem for Blending Quadrics}

Approaching the blending problem abstractly, we consider how to determine all degree 4 surfaces $S(F)$ that are tangent to two quadrics $S(G)$ and $S(H)$ at prescribed space curves. This problem was explored in detail in [4], and some preliminary considerations are needed before we can give it an exact formulation. Of course, we will assume that the quadrics 
$S(G)$ and $S(H)$ are not degenerate. That is, neither splits into a pair of planes.

Recalling Bezout's Theorem, a quartic surface intersects a quadric in a space curve of degree 8. If we require tangency, however, the curve in which the two surfaces are tangent must be counted double as it is the limit of two separate components, infinitesimally apart. In consequence, a quartic surface $S(F)$ is tangent to a quadric $S(G)$ in a curve $S(F, G)$ of degree 4. According to [9], the space curve $S(F, G)$ now must be of one of the following types:

(1) The curve $S(F, G)$ is irreducible but not planar, and so is the intersection of two quadrics.

(2) The curve $S(F, G)$ is irreducible and planar, and so is the intersection of a quartic surface and a plane.

(3) The curve $S(F, G)$ is reducible, and so is one of the following: Four lines, two lines and a conic, two conics, or one line and an irreducible degree 3 space curve.

We study the case when $S(F, G)$ is of type (1). $S(F, G)$ cannot be of type (2), since it then could not lie on $S(G)$ as planar curve of degree 4. When $S(F, G)$ is of type (3), we will need to catalogue a number of special cases, depending on the curve components. This curve type does arise, but neither its practical significance nor its mathematics has been sufficiently explored. With this in mind, we formulate the classification problem for blending quadrics with quartic surfaces as follows:

Given two irreducible quadric surfaces $S(G)$ and $S(H)$, and given on each an irreducible degree 4 space curve, $S\left(G, H^{\prime}\right)$ and $S\left(H, G^{\prime}\right)$ as the complete intersection with auxiliary quadric surfaces $S\left(H^{\prime}\right)$ and $S\left(G^{\prime}\right)$. Characterize the set of all quartic surfaces $S(F)$ where $S(F)$ is tangent to $S(G)$ in the curve $S\left(G, H^{\prime}\right)$ and $S(F)$ is tangent to $S(H)$ in the curve $S\left(H, G^{\prime}\right)$.

Note that the irreducibility of a space curve $S(G, H)$ in projective space implies that the degree 2 terms of $G$ and of $H$ are coprime polynomials. For if these polynomials are not coprime, then $S(G, H)$ has a planar component at infinity, hence is not irreducible [10].

We solve the above problem under the additional assumption that the two quadrics $S(G)$ and $S(H)$ have an irreducible intersection curve $S(G, H)$. That is, the curve $S(G, H)$ is of type (1) above. Moreover, we assume that the intersection curve $S(G, H)$ is different from $S\left(G, H^{\prime}\right)$ and from $S\left(H, G^{\prime}\right)$. Clearly this is the case for all blending surfaces considered in practice. 


\section{Completeness Theorem}

Let $S(G)$ and $S(H)$ be irreducible quadrics. Let $S\left(G^{\prime}\right)$ and $S\left(H^{\prime}\right)$ be two quadrics such that $S\left(G, H^{\prime}\right)$ and $S\left(H, G^{\prime}\right)$ are irreducible, and assume that $S(G, H)$ is irreducible and coincides neither with $S\left(G, H^{\prime}\right)$ nor with $S\left(H, G^{\prime}\right)$. Then every degree 4 surface $S(F)$ tangent to $S(G)$ in the curve $S\left(G, H^{\prime}\right)$ and tangent to $S(H)$ in the curve $S\left(H, G^{\prime}\right)$ has the equation

$$
K^{2}-\mu G H=0
$$

where $\mu$ is a constant and $S(K)$ is a quadric containing both $S\left(G, H^{\prime}\right)$ and $S\left(H, G^{\prime}\right)$.

The proof of this theorem in [4] proceeds as follows: Since $S\left(G, H^{\prime}\right)$ and $S\left(H, G^{\prime}\right)$ are irreducible and $S(F)$ contains these curves, the polynomial $F$ can be written as $F=A G+B^{\prime} H^{\prime}$ and as $F=B H+A^{\prime} G^{\prime}$, for some polynomials $A, A^{\prime}, B$, and $B^{\prime}$. These equations follow from Hilbert's Nullstellensatz. By considering the partial derivatives of $F$, the requirement of tangency to $S(G)$ and $S(H)$ in the curves implies further that $B^{\prime}=C H^{\prime}$ and $A^{\prime}=D G^{\prime}$, where $C$ and $D$ are some other polynomials. That is, we may write

$$
F=A G+C H^{\prime 2}=B H+D G^{\prime 2} \text {. }
$$

It would be a mistake to make a priori assumptions about the respective degrees of the polynomials $A, B, C$ and $D$. However, irreducibility of $S\left(G, H^{\prime}\right)$ and $S\left(H, G^{\prime}\right)$ in projective space has the consequence that that $A$ and $B$ must be polynomials of degree 2, and that $C$ and $D$ must be constants. The proof is combinatorial in nature, and makes use of the fact that the degree 2 terms of $G$ and $H^{\prime}$, as well as the degree 2 terms of $H$ and $G^{\prime}$ are coprime.

Having so deduced the form of the polynomial $F$, the difference polynomial $\left(A G+C H^{\prime 2}\right)-\left(B H+D G^{\prime 2}\right)$ is considered modulo the ideal $(G, H)$. Since this polynomial is zero, $C H^{\prime 2}-D G^{2}=0 \bmod (G, H)$. Moreover, since $C$ and $D$ are constants, the polynomial $C H^{\prime 2}-D G^{\prime 2}$ factors over the field of complex numbers. At this point the irreducibility of $S(G, H)$ enters into the proof, for it allows us to conclude, by primality of the ideal $(G, H)$, that one of the factors is in $(G, H)$, i.e., that it can be written $a G+b H$, where $a$ and $b$ are constants. Now it is simple to bring $F$ into the required form $F=K^{2}-\mu G H$.

The existence of the quadric $K$ is of considerable interest. For if the two curves of tangency $S\left(G, H^{\prime}\right)$ and $S\left(H, G^{\prime}\right)$ do not lie on a common quadric surface $S(K)$, then no quartic blending surface $S(F)$ will be found in general. This does not preclude the existence of special cases when one or several of the assumptions of the theorem do not hold. We will demonstrate such an exceptional case: 


\section{Example}

In [3], color plate 6 , a blending surface is shown that smoothly joins two elliptic cylinders. The two cylinders are given by $G=x^{2}+4 y^{2}-4$ and $H=9 x^{2}+y^{2}-9$. They intersect in four parallel lines, hence $S(G, H)$ is not irreducible and the Completeness Theorem does not apply. The blending surface has the equation

$$
F=\left(x^{2}+4 y^{2}-4\right)(z-3)^{2}+\left(9 x^{2}+y^{2}-9\right)(z+3)^{2}=0 .
$$

We show that $F$ cannot be of the form $K^{2}-\mu G H$, for any quadratic polynomial $K$.

We seek a quadratic polynomial $K$ such that $S(K)$ contains the curves of tangency $S(G, z+3)$ and $S(H, z-3)$, two ellipses in parallel planes. Clearly, $K=(z-3)(z+3)$ defines the only reducible quadric containing these curves. But $\alpha K^{2}+\beta G H$ contains no $x^{2} z^{2}$ term, so that $K=(z-3)(z+3)$ does not qualify.

Next, let us assume that an irreducible quadric contains the curves $S(G, z+3)$ and $S(H, z-3)$. Since $S(K)$ contains $S(G, z+3)$, we have $K(x, y,-3)=\alpha G$, for some $\alpha \neq 0$. Without loss of generality we assume $\alpha=1$ and conclude that $K=x^{2}+4 y^{2}+z K_{1}$, where $K_{1}$ is linear. Next, the containment of $S(H, z-3)$ implies $K(x, y, 3)=\beta H$ for $\beta \neq 0$. Comparing the $x^{2}$ and $y^{2}$ terms on both sides, we have $x^{2}=9 \beta x^{2}$ and $4 y^{2}=\beta y^{2}$. Hence there is no irreducible quadric containing both $S(G, z+3)$ and $S(H, z-3)$. It follows that $F$ cannot have the form $G H-\mu K^{2}$.

In principle, the Completeness Theorem provides a constructive procedure for deriving blending surfaces, as follows:

(1) Given the quadrics $S(G)$ and $S(H)$, pick a quadric $S(K)$ intersecting the other two surfaces in the desired curves of tangency.

(2) Pick a parameter $\mu$ and set $F=K^{2}-\mu G H$. Then $S(F)$ is a blending surface.

After so determining $F$, only the area between the curves of tangency is of interest as blending surface. Consequently, $S(F)$ must be suitably clipped. We will see later how this is done with the help of $S(K)$.

How should $K$ and $\mu$ be selected? Picking $S(K)$ obviously specifies the curves at which we tangentially connect the blending surface to the primary surfaces. The numerical parameter $\mu$ then controls the curvature of the resulting blending surface. However, considerable experience is necessary before a designer can skillfully manipulate blending surfaces so derived. Therefore, we explore alternative formulations. 


\section{Affine Parameter Space Derivations}

The blending surfaces characterized above can be derived by substitution from a parametric base curve, as we now explain. The resulting derivation procedure is called the potential method [3]. According to whether the base curve is viewed in affine or projective space, we speak of the affine or the projective potential method, respectively. We now explain the affine potential method.

The potential method derivation has the advantage that a greater intuitive understanding of the resulting shapes is obtained. Moreover, it becomes clear that this formulation can be used to blend arbitrary algebraic surfaces, and that higher order continuity to the primary surfaces could be achieved should the application require it.

A simple introduction to the parameter space formulation is provided by a twodimensional example. Consider the circle specified by the polynomial $f(s, t)=(s-1)^{2}+(t-1)^{2}-1$ in the $s, t$-plane. This circle may be considered a blending curve for the intersecting coordinate axes $t=0$ and $s=0$, as shown in Figure 4.1.

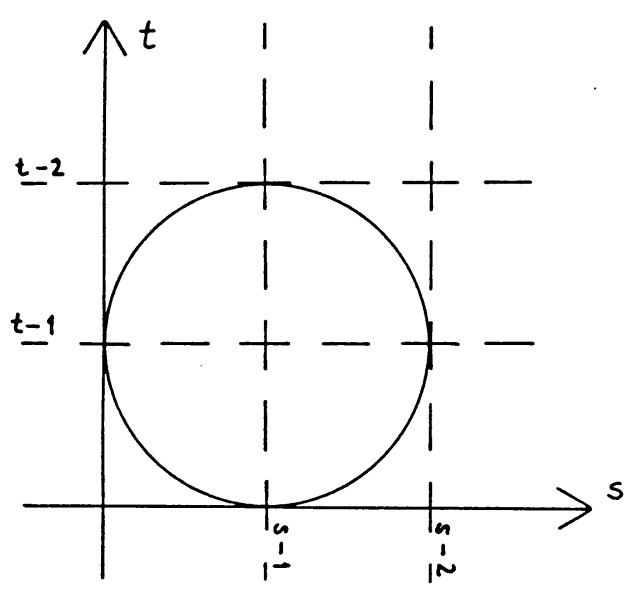

Figure 4.1

In analogy to the three dimensional case, denote the circle by $S(f)$, the $s$-axis by $S(t)$, and the $t$-axis by $S(s)$. Substitute the polynomial $G(x, y)$ for $s$, and the polynomial $H(x, y)$ for $t$. Then $f$ becomes the polynomial $F=(G-1)^{2}+(H-1)^{2}-1$. What is the shape of $S(F)$ in $x, y$ space? The answer is illustrated in Figure 4.2 where the circles $S\left(x^{2}+y^{2}-1\right)$ and $S\left((x-1)^{2}+y^{2}-1\right)$ have been used. Intuitively, we have redrawn $S(f)$ in a curvilinear coordinate system. Moreover, we note that $S(F)$ remains tangent to $S(G)$ and $S(H)$. Since $G$ and $H$ have degree $2, F$ has degree 4 . 


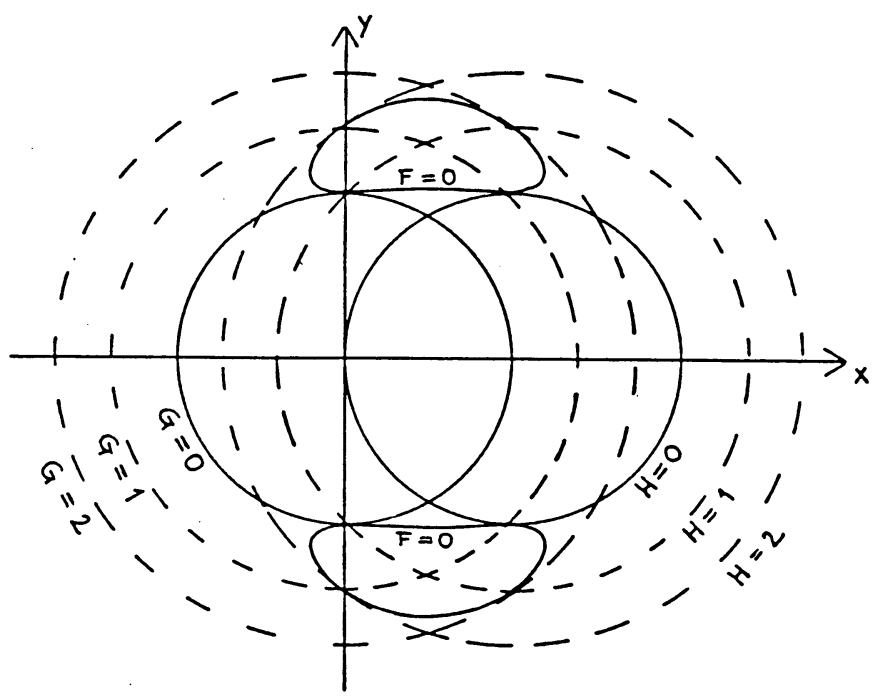

Figure 4.2

Consider the point $(u, v)$ on $S(f)$. This point is the intersection of the lines $S(s-u)$ and $S(t-v)$. Corresponding to $(u, v)$ in $s, t$-space are all points of the intersection of $S(G-u)$ with $S(H-v)$, in $x, y$-space. We therefore can think of the substitution for $s$ and $t$ as a deformation of the coordinate system. Note, however, that different points in the curved $x, y$-space may have the same coordinates $(G-u, H-v)$, as is the case in Figure 4.2.

We embed $s, t$-space into three dimensional $r, s, t$-space. Now $S(s)$ and $S(t)$ are planes, and $S(f)$ is a circular cylinder that is tangent to these planes in the lines $S(s, t-1)$ and $S(s-1, t)$, respectively. If $G$ and $H$ specify surfaces in $x, y, z$-space and $F$ is obtained from $f$ through substitution as before, then $S(F)$ will be a surface tangent to $S(G)$ and $S(H)$. In particular, $S(F)$ is tangent to $S(G)$ in $x, y, z$-space in the curve $S(G, H-1)$.

Intuitively, the reason tangency is preserved can be understood as follows: A transformation through substitution maps intersection points to intersection points. Now tangency is a double intersection, hence tangency is preserved. A formal proof is easily found. Briefly, the partial derivatives of $F$ on the curves $S(G, H-1)$ and $S(H-1, G)$ must agree with the corresponding derivatives of $G$ and $H$. It is then easy to see that the tangent planes must coincide along the curves; see also [3].

We extend the underlying parameter function $f$ to include all conic cylinders tangent to $S(s)$ and $S(t)$. Assuming tangency at $S(s-a, t)$ and $S(s, t-b)$, where $a$ and $b$ are given constants, all such conic cylinders are given by

$$
f=b^{2}(s-a)^{2}+a^{2}(t-b)^{2}-a^{2} b^{2}-2 \lambda a b s t
$$

Here $\lambda$ is a number that should be greater than -1 . In the range $-1<\lambda<1$ we obtain an 
elliptic cylinder, for $\lambda=1$ a parabolic cylinder, and for $\lambda>1$ a hyperbolic cylinder. See also Figure 4.3.

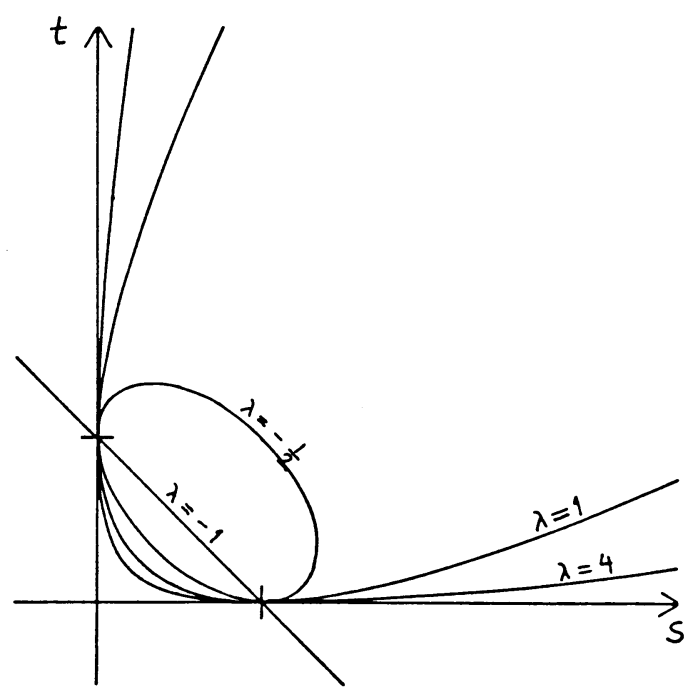

Figure 4.3

For $\lambda=-1$ the cylinder degenerates into the double plane $S\left((b s+a t-a b)^{2}\right)$. For $\lambda<-1$ we obtain hyperbolic cylinders that are positioned as shown in Figure 4.4. Due to their position, the hyperbolic cylinders for $\lambda<-1$ are not useful for deriving blending surfaces.

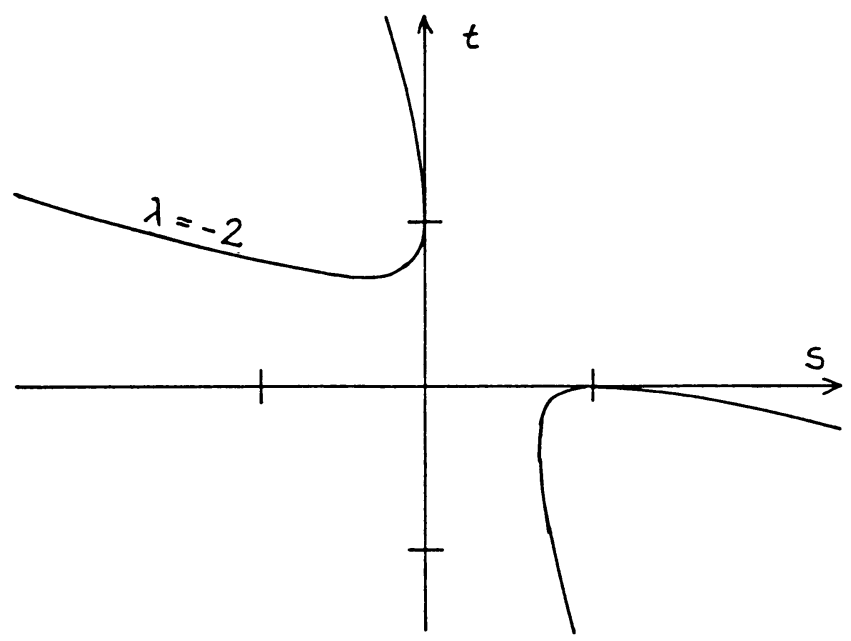

Figure 4.4

Substituting quadric surfaces $S(G)$ and $S(H)$ into this family of cylinders yields quartic blending surfaces. The relationship to the normal form $F=K^{2}-\mu G H$ derived previously for blending quadrics is seen directly from the equation for $f$ when it is rewritten as

$$
f=(b s+a t-a b)^{2}-2(1+\lambda) a b s t
$$

Hence $S(K)$ is the image of the plane $S(b s+a t-a b)$, and $\mu=2(1+\lambda) a b$. Referring again to Figure 4.3 , it is easy to see that the plane $S(b s+a t-a b)$ can be used to identify the part of $S(F)$ that is used for blending: Clip those points of $S(f)$ for which $b s+a t-a b>0$, or, 
equivalently, clip $S(F)$ when $K(x, y, z)>0$. This will suffice for all elliptic and parabolic base cylinders, but for the hyperbolic cylinders the other branch of the hyperbola must be clipped when $s<0$ or when $t<0$, or, equivalently, $S(F)$ is clipped when $G<0$ or when $H<0$.

We see now that blending surfaces may be derived by substituting the primary surfaces to be blended in the parametric polynomial $f$. Intuitively, this is a deformation of the coordinate system in which the coordinate planes $S(s)$ and $S(t)$ are mapped to the primary surfaces $S(G)$ and $S(H)$. The conic cylinder $S(f)$ blending the planes is mapped to the surface $S(F)$ blending $S(G)$ and $S(H)$. Finally, the plane $S(b s+a t-a b)$ is mapped to the surface $S(K)$, familiar from the normal form equation for quartic blends. This surface $S(K)$ plays an important role both in defining the curves of tangency, as well as in providing the criterion for clipping $S(F)$.

The blending method described works equally well for blending surfaces of degree higher than 2. No intrinsic property of quadrics is needed to prove tangency of the resulting surfaces $S(F)$, given the primary surfaces $S(G)$ and $S(H)$ [3]. Moreover, one may use other parametric polynomials $f$. In [5] we have advocated quadratic polynomials for the simple reason that the resulting blending surfaces $S(F)$ have a small degree. If degree is of no concern, or if higher order continuity to the primary surfaces is needed, higher degree polynomials $f$ may be used. For example, [7] has used the super ellipses as a parametric base polynomial.

It is not necessary to think of a three dimensional parameter space. In [3], a two dimensional $s, t$-space is used instead, and an intuitive interpretation of the substitution process given. However, for other purposes such as corner blending the three dimensional view seems more natural.

Clearly, the substitution paradigm yields a very intuitive derivation method, and for quadratic base polynomials $f$ the quantities $a, b$, and $\lambda$ are easily understood. Briefly, the choice of $a$ and $b$ controls the distance the respective curves of tangency on $S(H)$ and $S(G)$ have, from the intersection curve $S(G, H)$. Enlarge $a$ for example, and the curve at which $S(F)$ is tangent to $S(H)$ is further apart from $S(G, H)$. Of course, for a closed quadric surface $S(H)$ a maximum distance cannot be exceeded. Moreover, the choice of $\lambda$ controls the "sag" of the blending surface, i.e., how closely it follows $S(G) \cup S(H)$. [5] contains a number of pictures illustrating the choice of $\lambda$.

The substitution paradigm with a quadratic base polynomial $f$ provides a very intuitive procedure for deriving blending surfaces given $G$ and $H$. At least in the case of 
quadrics it is easy to visualize the behavior induced by choosing $a, b$ and $\lambda$. Little additional work seems to be needed to automate the method and incorporate it into a solid modeler. Difficulties to be faced for blending higher degree primary surfaces $S(G)$ all have to do with understanding the shape of the surfaces $S(G-s)$ when $0<s \leq a$. For quadrics it is easy to see how these surfaces behave, with the cone perhaps exhibiting the most complex behavior. For higher degree surfaces this relationship may be much more complex, and without a comprehensive surface classification an automated blending surface derivation appears to be difficult.

\section{Projective Parameter Space Derivation}

The affine potential method described in the previous section is not general enough to derive all quartic blends of the form $K^{2}-\mu G H$. The limitation is due to the fact that we formulated the derivation based on affine polynomials $f$. Only by using a projective base polynomial $f$ and substituting for the homogenizing variable $u$ as well do we obtain the full generality possible. We now describe the projective potential method.

Consider a projective parameter space with homogeneous coordinates $r, s, t$, and $u$. The quadratic base polynomial is then

$$
f_{1}=b^{2}(s-a u)^{2}+a^{2}(t-b u)^{2}-a^{2} b^{2} u^{2}-2 \lambda a b s t
$$

As before, we substitute $G$ for $s$ and $H$ for $t$. However, we now choose a polynomial $W$ to be substituted for $u$. It is reasonable to limit the degree of $W$ by the maximum degree of $G$ and $H$. Thus, when blending quadrics, we substitute a polynomial of degree no higher than 2 thereby obtaining quartic blending surfaces.

This projective method of deriving blending surfaces subsumes the affine method, since by substituting the polynomial $W=1$ for $u$ the affine method is recovered. This is to be expected, for it corresponds to the usual way of embedding affine space into projective space.

Before exploring the generality of the projective derivation procedure, we seek to simplify the base polynomial. It is well known that in projective space all nondegenerate conics are related by projective transformation. In particular, the base polynomial $f_{1}$ above can be transformed into the polynomial

$$
f_{0}=\left(s^{\prime}-u^{\prime}\right)^{2}+\left(t^{\prime}-u^{\prime}\right)^{2}-u^{\prime 2}
$$

by the projective transformation 


$$
\begin{gathered}
r=r^{\prime} \\
s=\frac{s^{\prime}}{b c} \\
t=\frac{t^{\prime}}{a c} \\
u=\frac{(1-c) s^{\prime}}{a b c}+\frac{(1-c) t^{\prime}}{a b c}+\frac{u^{\prime}}{a b}
\end{gathered}
$$

where $c=\sqrt{1+\lambda}$. For $\lambda=-1$ the conic degenerates, and the projective transformation becomes singular. We again assume that $\lambda>-1$, hence $\sqrt{1+\lambda}$ is real.

We claim that the choice of $W$ already includes the role of $a, b$, and $\lambda$. That is, let $F_{1}$ be the result of substituting $G, H$ and $W$ for $s, t$ and $u$ in $f_{1}$ above. Then we can find a polynomial $W^{\prime}$ such that substituting $G, H$ and $W^{\prime}$ for $s^{\prime}, t^{\prime}$ and $u^{\prime}$ in $f_{0}$ we obtain $F_{0}$ such that $S\left(F_{1}\right)=S\left(F_{0}\right)$. To see this, we rewrite the polynomials as

$$
\begin{gathered}
f_{1}=(b s+a t-a b u)^{2}-2(1+\lambda) a b s t \\
f_{0}=(s+t-u)^{2}-2 s t
\end{gathered}
$$

Let $W^{\prime}=(a b W-b G-a H) / \sqrt{a b(1+\lambda)}+G+H$. Substituting $G, H$ and $W^{\prime}$ for $s^{\prime}, t^{\prime}$ and $u^{\prime}$ in $f_{0}$ we obtain

$$
F_{0}=(b G+a H-a b W)^{2} /(a b(1+\lambda))-2 G H
$$

Since substitution of $G, H$ and $W$ for $s, t$ and $u$ in $f_{1}$ yields

$$
F_{1}=(b G+a H-a b W)^{2}-2 a b(1+\lambda) G H
$$

we have $S\left(F_{1}\right)=S\left(F_{0}\right)$, unless $\lambda=-1$. Hence for nondegenerate conics the projective base polynomial $f_{0}$ suffices.

Now consider a quartic blending surface $S(F)$ where $F=K^{2}-\mu G H, S(G)$ and $S(H)$ are quadrics, and $K$ is of degree 2 or less. Then with $W=G+H-K / \sqrt{\mu}$ we have $S(F)=S\left(F^{\prime}\right)$, where $F^{\prime}$ is obtained from $f_{0}$ by substituting $G, H$, and $W$. Hence every quartic surface obtained through the methods of Section 3 is also derivable from $f_{0}$ using the projective derivation method.

Although based on substitution as in the case of the affine derivation method, the role of $W$ is difficult to visualize. More work is needed to interpret how the form of $W$ influences the shape of the resulting blending surfaces, and automating the projective procedure appears difficult. A specific difficulty arises from the form of polynomials as follows: Given $\alpha \neq 0$, the surfaces $S(G)$ and $S(\alpha G)$ are clearly equal. Yet using $\alpha G$ in place of $G$ when substituting alters the resulting blending surface. 


\section{Projective Transformation of Blending Surfaces}

We investigate the behavior of the blending surface $S(F)$ under projective transformation of the projective $x, y, z, w$-space. In particular, we settle the question when a projectively derived blending surface can be obtained by first deriving an affine blending surface followed by a projective transformation of $x, y, z, w$-space. As in Section 3 , we concentrate on quartic blends of quadrics.

Since we embed the blending surfaces into complex projective space, we assume throughout that all polynomials $F, G, H$, etc., are homogeneous in $x, y, z$, and $w$. Because of the results of the previous section, we make the following definition:

\section{Definition}

A blending surface $S(F)$ with $F=K^{2}-2 G H$ is projective if it is obtained from $f_{0}=(s+t-u)^{2}-2 s t$ by substituting $G$ for $s, H$ for $t$, and $W$ for $u$. Moreover, if $W=\alpha w^{2}$, $\alpha \neq 0$, then $S(F)$ is an affine blending surface.

First, let us exclude substituting the zero polynomial for $u$, for it leads to a degenerate blending surface: If $W=0$ is substituted into $f_{0}$, then $F=G^{2}+H^{2}$, hence $F=(G+\mathrm{i} H)(G-\mathrm{i} H)$, where $\mathrm{i}=\sqrt{-1}$. Since $F$ factors, it is in general a surface that intersects $G$ and $H$ transversally, i.e., it is useless as a blending surface.

Given a projective blend $S\left(F_{0}\right)$ of the quadrics $S\left(G_{0}\right)$ and $S\left(H_{0}\right)$, is there a projective transformation $p$ such that $S(F)$ is the image of an affine blend $S(F)$, i.e., $F_{0}=p(F)$ ? We solve this problem with the following theorem:

Theorem

Let $S\left(F_{0}\right)$ be a projective blend where $F_{0}=K_{0}^{2}-2 G_{0} H_{0}$, and $G_{0}, H_{0}$, and $K_{0}$ are quadratic. Let $p$ be a projective transformation such that $p(F)=F_{0}, p(G)=G_{0}, p(H)=H_{0}$, and $p(K)=K_{0}$. If $F$ is an affine blending surface, then $W_{0}=G_{0}+H_{0}-K_{0}=U^{2}$, for a linear form $U$. Conversely, if $W_{0}=G_{0}+H_{0}-K_{0}=U^{2}$, then $p$ may be chosen such that $F$ is an affine blending surface.

Proof: Assume $F$ is an affine blend, so that $F$ is obtained from $f_{0}$ via the substitution $s=G, t=H, u=G+H-K=\alpha w^{2}$. Let $\beta=\sqrt{\alpha}$. The transformation $p$ effects a substitution $w=a x^{\prime}+b y^{\prime}+c z^{\prime}+d w^{\prime}=U$. Since $p$ is not singular, $U$ is not the zero polynomial. Then

$$
p(G+H-K)=p(G)+p(H)-p(K)=(\beta U)^{2} .
$$

Moreover, since $F=K^{2}-2 G H$, we have $p(F)=F_{0}=p(K)^{2}-p(G) p(H)$. With $G_{0}=p(G)$, $H_{0}=p(H), K_{0}=p(K)$, and with $W_{0}=(\beta U)^{2}$ the projective blend has the required structure. 
Conversely, assume that $F_{0}$ is a projective blend with $W_{0}=G_{0}+H_{0}-K_{0}=U^{2}$. Let $U=a x^{\prime}+b y^{\prime}+c z^{\prime}+d w^{\prime}$. If $d \neq 0$, consider the transformation $q$ given by $x^{\prime}=x, y^{\prime}=y, z^{\prime}=z$, and $w^{\prime}=(w-a x-b y-c z) / d$. Then $q\left(W_{0}\right)=q(U)^{2}=w^{2}$. Since $W_{0}=G_{0}+H_{0}-K_{0}$, we have $q\left(F_{0}\right)=q\left(G_{0}+H_{0}-W_{0}\right)^{2}-2 q\left(G_{0}\right) q\left(H_{0}\right)$. Consequently, $q\left(F_{0}\right)$ is an affine blend. If $d=0$ we may assume without loss of generality that $a \neq 0$. We take as $q$ the transformation $x^{\prime}=(w-c y-b x) / a, y^{\prime}=x, z^{\prime}=y, w^{\prime}=z$. Then $q\left(W_{0}\right)=w^{2}$. The theorem follows with $p$ the inverse transformation of $q$.

It is interesting to note that not all projective blending surfaces are the image of affine blending surfaces. Rather, the proof of the theorem suggests classifying the quartic blends $S\left(K^{2}-2 G H\right)$, based on the form of $W=G+H-K$. This classification is precisely the projective classification of quadrics [1], already mentioned in Section 2:

(1) $W=x^{2}+y^{2}+z^{2}+w^{2}$, the imaginary sphere.

(2) $W=x^{2}+y^{2}+z^{2}-w^{2}$, the real sphere.

(3) $W=x^{2}+y^{2}-z^{2}-w^{2}$, the one-sheeted hyperboloid.

(4) $W=x^{2}+y^{2}+w^{2}$, the imaginary cylinder.

(5) $W=x^{2}+y^{2}-w^{2}$, the real cylinder.

(6) $W=x^{2}+w^{2}$, two imaginary planes.

(7) $W=x^{2}-w^{2}$, two real planes.

(8) $W=w^{2}$, the double plane.

The last class consists of all affine blends and the projective blends characterized by the theorem. The other classes can be elaborated similarly.

Note that purely imaginary surfaces are of practical interest as $W$. For example, we may blend two intersecting cylinders as follows: Let $G=2 x^{2}+2 y^{2}-2 w^{2}, H=2 y^{2}+2 z^{2}-2 w^{2}$, and $W=x^{2}+y^{2}+z^{2}+w^{2}$. The resulting blending surface is tangent at the intersection of the cylinders with $K=x^{2}+3 y^{2}+z^{2}-3 w^{2}$, and does not have the undesirable "bulge" near the points $(0,1,0,1)$ and $(0,-1,0,1)$ seen in the illustration in [6]. This bulge cannot be avoided for affine blending surfaces, i.e., when using $W=w^{2}$.

\section{Other Work and Conclusions}

Perhaps the most natural class of blending surfaces are the canal surfaces [8]. These surfaces are obtained as envelope of the volume swept by a moving sphere of fixed radius 
that is kept in contact with both primary surfaces to be blended. There are major difficulties associated with this approach:

(1) Canal surfaces typically are of high algebraic degree. For example, the canal surface blending two circular cylinders intersecting at right angle has degree 16 in the simplest case.

(2) Generic derivation of canal surface equations is computationally very expensive. Since the surface degree is high and since a generic formulation must make explicit the dependency on shape parameters, very complicated symbolic forms are needed. Expressions with several thousand terms are not uncommon.

(3) As the radius of the moving sphere is increased in an attempt to obtain a broader blending surface, unexpected self intersections may result and a surface with creases may be obtained.

In [8], Rossignac and Requicha give a method to approximate canal surface blends with torus and cylinder segments. By approximating the canal surface, the degree and derivation problems are nicely side stepped. However, while the segments join each other smoothly, the resulting surface is not tangent to curved primary surfaces to be blended. The method is used in a CSG modeler with standard primitives, and much of the charm of the work derives from the fact that the underlying set of primitives needs no extension.

In [6], Middleditch and Sears outline a blending method remarkably similar to the affine potential method. The method is based on conics tangent to coordinate lines in affine $s, t$ parameter space. The conics used are restricted to be tangent at points equidistant from the coordinate lines, i.e., $a=b$ is assumed. Moreover, our parameter $\lambda$ is expressed as distance of the apex of the conic from the lines. Since Middleditch and Sears seek to derive blending surfaces for which the curves of tangency lie at a fixed Euclidian distance from the other surface, they must substitute complicated offset equations into the underlying conic. In consequence, fairly complex blending surface equations are obtained. For instance, blending a cone and a plane results in a surface of degree 6. Middleditch and Sears observe that substituting the implicit equations of the surfaces to be blended leads to algebraically simpler surfaces. This class of simpler blending surfaces is a subset of the affine blending surfaces. It is a proper subset since $a=b$ is assumed for the underlying conic. The paper also discusses in detail how to integrate the blending method into a CSG modeler with standard primitives. 
Rockwood and Owen give a blending method in [7] that is also based on substitution. The basic curve used is the super ellipse

$$
(1-s / a)^{\lambda}+(1-t / b)^{\lambda}=1
$$

where the parameters $a, b$, and $\lambda$ are analogous to the parameters in the affine potential method. The surfaces to be blended are substituted for $s$ and $t$. The constants $a$ and $b$ can be replaced with functions of the gradients of the primary surfaces. This seems to have the effect of making the curvature distribution more uniform along the length of the blending surface. When $\lambda>2$, or when gradient functions are used in place of the constants $a$ and $b$, blending surfaces of fairly high algebraic degree are obtained. Moreover, for large values of $\lambda$ the order of continuity of the blend with the primary surfaces increases. In both cases numerical difficulties should be expected.

The methods of $[6,7,8]$ each have been incorporated into some experimental solid modeling systems. This requires some degree of automating the blending process whose prospects we now discuss briefly. Our insights are limited by the fact that so far we have not had the opportunity to experiment with these systems.

Two problem types are encountered when incorporating a blending method into a solid modeling systems:

(1) Local control problems: The blending surface(s) to be interpolated must be specified in an intuitive and concise manner that delivers predictable results anticipated by the user. This requires an intuitive interpretation of all parameters in the underlying polynomial.

(2) Blend-blend problems: When blending edge cycles bounded by different faces, a contiguous blending surface can only be piecewise algebraic. This not only complicates blending in that smooth transitions between the pieces are required, but also introduces additional complexities for clipping. In particular, in CSG type modelers blending operations must be analyzed for sequence dependency which must be understood fully by the user.

All authors have acknowledged the existence of the two problem types, but concentrate mostly on blend-blend problems. Perhaps this is because these problems must be faced immediately by any implementation.

In the case of [8], it appears reasonable to concentrate on blend-blend problems, since the only parameter that is specified for local shape control is the radius of the moving sphere. Nevertheless, additional results would be desirable that provide information about 
the precision to be expected when choosing the number of primitive segments with which the canal surface is to be approximated. For instance, when blending two intersecting circular cylinders of radius 10 with a canal surface of radius 1 , what precision can be expected from an approximation of, say, 100 segments of tori and cylinders?

The blending method of [6] aims at solving the local control problem by giving the base polynomial a restricted form and explicitly linearizing distance control parameters. However, such a formulation shifts the problem to finding suitable potential functions that conform with the shape expectations based on Euclidian distance measures. When the set of underlying primitives is simple and fixed, this appears to be reasonable. However, the potential functions needed to blend blending surfaces recursively, e.g., when blending at corners, quickly complicate the problem, as acknowledged by the authors.

Also in [7] the local control problem remains largely unexplored. One is left with the impression that the user must determine the desired shape parameters interactively, perhaps by inspecting the resulting surfaces visually. However, [7] also suggests a blending method similar to the homotopy technique we have outlined in [3] for which the local control problem seems more tractable.

All blending methods based on substitution face the difficulty that the deformation effected by this substitution has very few intrinsic properties that would allow solving local control problems in general. Therefore, we believe that the geometric properties of blending surfaces for specific surface classes must be explored first, e.g., for the class of all quadrics, or for the class of all CSG primitives. Clearly, these properties must be fully understood and interpreted in the context of the primitive class considered before the method should be automated. Only then is it possible to give to the casual user a clear understanding of the range of specification choices and their results.

\section{References}

(1) W. Blaschke

"Projektive Geometrie", Birkhaeuser, Stuttgart, West Germany, 1954.

(2) W. Fulton

"Algebraic Curves", W. A. Benjamin, New York, 1969. 
(3) C. Hoffmann and J. Hopcroft

"Automatic Surface Generation in Computer Aided Design", The Visual Computer 1:2, Springer Verlag, 1985, 92-100.

(4) C. Hoffmann and J. Hopcroft

"Quadratic Blending Surfaces", Computer Aided Design 18, July 1986.

(5) C. Hoffmann and J. Hopcroft

"The Potential Method for Blending Surfaces and Corners", in Geometric Modeling, G. Farin, ed., SIAM Publishing; to appear Fall 1986.

(6) A. Middleditch and K. Sears

"Blend Surfaces for Set Theoretic Volume Modeling Systems", SIGGRAPH Comp. Graphics 19:3, 1985, 161-170.

(7) A. Rockwood and J. Owen "Blending Surfaces in Solid Geometric Modeling", in Geometric Modeling, G. Farin, ed., SIAM Publishing; to appear Fall 1986.

(8) J. Rossignac and A. Requicha

"Constant-Radius Blending in Solid Modeling", Comp. in Mech. Engr. 3,1984, 65-73.

(9) V. Snyder and C. Sisam

"Analytic Geometry of Space", H. Holt, New York, 1914.

(10) J. Warren

Oral communication, 1985. 